\title{
Repair of $\gamma$-ray-induced base damage in L5178Y sublines is damage type-dependent and unrelated to radiation sensitivity ${ }^{*}$
}

\author{
Marcin Kruszewski ${ }^{1,2 \Omega}$, Tomasz H. Zastawny ${ }^{3}$ and Irena Szumiel $^{1}$
}

${ }^{1}$ Department of Radiobiology and $\mathrm{H}$ ealth Protection, Institute of N uclear Chemistry and Tech nol ogy, W ar szawa, Poland; ${ }^{2}$ Department of ExperimentalH ematology and Cord Blood Bank, M. Sk3odowska-Curie M emorial Can cer Cen ter and Institute of Oncology, W arszawa, Poland; ${ }^{3}$ Department of Clin i cal Biochem istry, University School of Medical Sciences, Bydgoszcz, Poland

Received: 31 J anuary, 2000; revised: 2 February, 2001; accepted: 20 March, 2001

Keywords: $\gamma$-rays, DNA base dam age re pair, ra di a tion sen si tivity, L5178Y murinelymphoma sublines

The L5178Y (L Y) murinelym phoma sublines $L Y-R$ and $L Y-S$ are dif fer en tially sen si tive to ion iz ing ra di a tion. The high ra di a tion sen si tivity of $L Y-S$ cells is re lated to impaired rejoining of DNA double strand breaks. We found previously that the $\gamma$-ray-induced base dam age is higher in the more radiosensitive $L Y$-S subline. Here, we ex am ine therole of there pair of ion iz ingra di a tion in duced basedam agein rela tion to the radiosensitivity dif fer ence of these sublines.

We used the GS/MS tech nique to es ti mate the re pair rates of six types of base damage in $\gamma$-irradiated LY cells. All mod i fied DNA bases iden ti fied in the course of this study were typ i cal for ir ra di ated chromatin. The to tal amount of ini tial base dam age was higher in the ra di a tion sen si tive $L Y-S$ subline than in the ra di a tion re sistant $L Y-R$ subline. The re pair rates of 5-0 H MeU ra, 5-0 HCyt, 8-0 H Ade were sim i lar in both cell lines, the re pair rates of $F$ apyAde and 8-OH Gua were higher in the radiosensitive $L Y-S$ cell line, whereas the re pair of 5-OH U ra was faster in its radio resistant coun ter part, the LY-R.

Al to gether, the re pair rates of the $\gamma$-ray-induced DNA base dam age in LY sublines are re lated nei ther to the ini tial amounts of the dam aged bases nor to the dif fer en tial le thal or mutagenic ef fects of ion iz ing ra di a tion in these sublines.

\footnotetext{
This work was partly sup ported by the State Com mitteefor Sci en tific Research (KBN) (stat u tory INCT grant, MK and IS) and by KBN Grant 4 P05A.121.08 (THZ and MK).

$\Omega^{\Omega}$ Cor responding au thor: Marcin K ruszewski, In sti tute of N u clear Chem is try and Tech nol ogy, Dorodna 16, 03-195 Warszawa, Po land; tel: (48 22) 811 0736, fax: (48 22) 811 1532, e-mail: marcinkr@or ange.ichtj.waw.pl

Abbreviations: 5-OHHyd, 5-hydroxyhydantoin; 5-OHCyt, 5-hydroxycytosine; 5-OHMeU ra, 5-hydroxymethyluracil; 5,6-diOHUra, 5,6-dihydroxyuracil; FapyAde, 4,6-diamino-5-formamidopyrimidine; 80HAde, 8-hydroxyadenine; 8-OHGua, 8-hydroxyguanine; BSTFA, bis(trimethylsilyl)-trifluoroacetamide; DSB, double strand break; GC/MS, gas chromatography/mass spectrometry.
} 
I onizing radiation-induced DNA lesions are locally clustered [1]. As reviewed by Wallace [2], when clus tered base dam age is pro cessed by base excision repair, a DNA dou ble strand break (DSB) can re sult. Logically, the de layed rejoin ing of DSB that is ob served in some cell lines can be caused by the low ef fi ciency of the DSB repair sys tem and/ or by DSB gen er ation during the post-irradiation period, as indicated by $W$ allace [2].

The aim of this study was to in vestigatethe repair of base damage induced by $\gamma$-rays in two related cell sublines dif fer ing in the sen sitivity to oxidative stress. The respective L5178Y sublines, LY-S and LY-R, display a unique fea ture of in versecross-sensitivity to $X$ rays and hydrogen peroxide [3-7]. The high sen si tiv ity of $L Y-S$ cells to $X$ rays $\left(D_{0}=0.5 \mathrm{~Gy}\right)$ is ex plained by theim pair ment of DSB rejoining [8] and high initial DNA damage [9-11]. In the case of hydrogen peroxide treatment the reasons for the enhanced sensitivity of LY -R cells are more com plex. These are: a less ef fi cient an ti oxi dant defencesystem [5], anda higher content of iron ions (available for entering the Fenton reaction [12] and generating the dam ag ing hydroxyl rad i cals). Hence, a signif i cantly higher amount of ini tial DNA le sions than that in LY -S cells $[6,13]$.

Thein duc tion of base dam age in $\gamma$-irradiated or hy dro gen per ox ide-treated LY sublines has been described previously; the extent of the initial damage was found to be related to the subline's sensitivity to the damaging agent [13]. In ter est ingly, a sim i lar relation to sen sitiv ity was described by M ori \& Dizdaroglu [14] for the parent L5178Y line and its radiosensitive mu tant M 10.

We undertook base damage determination by gaschromatography -mass spectrometry, which allowed us to discern and quantitate various base damage types. This, how ever, is only possible after irradiation with a supralethal dose (400 Gy). The en zy matic re pair activity that is detectable after such a massive dose gives a good reason to assume that it also is functional af ter ir radi a tion with lower doses. With the dif fer ent end-points examined previously (survival, DNA strand break induction and repair, mutation frequency) and in this re port, it is un avoid able to use a broad range of ra di a tion doses in or der to obtain an optimal damage range for each method. Such a dis crep ancy in the dose range ap plied pre vi ously and in this study seems to be acceptable, as we compare relative responses in the two cell sublines rather than absoluterelationsbetween damageesti mated at the molecular, subcellular and cellular levels.

Al though it is not pos si bleto di rectly dis cern between the pri mary and sec ond aryDSBs, examination of repair of the ionizing radiation-induced base dam age in $L Y$ sublines may give some indication as to the role of base dam age in the delayed re pair of DSBs in LY -S cells.

\section{MATERIALS AND METHODS}

Chemicals. Triton X-100 was purchased from Sigma Chemical Company. Internal stan dards were a gift from Dr. M. Dizdaroglu from the National Institute of Standards and Technology (Gaithersburg, MD, U.S.A.). Acetonitrile and bis(trimethylsilyl)-trifluoroacetamide (BSTFA) containing 1\% trimethylchlorosiliane were obtained from Pierce Chemical Co. Formic acid was from Mallincrodt.

Cell cultures. Murine leukaemic lymphoblasts $L Y-R$ and $L Y-S$ were main tained in suspension cul tures in Fischer's me dium sup ple mented with $8 \%$ bo vine se rum, as described by Szumiel [15]. Asynchronous populations in exponential phase of growth were used in all experiments.

Irradiation. Cells were collected by centrifugation and re sus pend ed in cold Fisher's me dium con tain ing $8 \%$ bo vinefoetal serum $(4 \times$ $10^{6}$ cells $/ \mathrm{ml}$ ). ${ }^{60}$ Co $\gamma$ rays were applied in an icebath, at a dose rate of $39.2 \mathrm{~Gy} / \mathrm{min}$ (MINEOLA, INCT), as previously described 
[13]. After irradiation, cell suspension aliquots were placed at $37^{\circ} \mathrm{C}$ for repair peri ods rang ing from 15 to $360 \mathrm{~min}$ and than frozen in liquid nitrogen and stored at $-80^{\circ} \mathrm{C}$ until chromatin isolation. To avoid artifactual oxidation of chromatin from dead cells, the cell membrane integrity was monitored by the nigrosine test. Throughout the whole repair period more than $90 \%$ of irradiated cells had cell membrane not permeable to nigrosine.

Iso la tion of chromatin and base dam age determination. This was carried out as described previously [13]. In brief, chromatin was iso lated ac cord ing to the mod i fied pro ce dure of M ee $\&$ Adelstein [16]. Chromatin samples con tain ing $100 \mu \mathrm{g}$ of DNA (as deter mined by spectrophotometry) were supplemented with internal standards, lyophilized and hydrolysed with $0.5 \mathrm{ml}$ of $60 \%$ formic acid in evacuated and sealed tubes for $30 \mathrm{~min}$ at $140^{\circ} \mathrm{C}$. The hy droly sates were lyophilized and then trimethylsilylated in polytetrafluoroethylene-capped hypovials (PierceChem i cal Co.) with $100 \mu \mathrm{l}$ of a mixture of BSTFA and acetonitrile $(4: 1, \mathrm{v} / \mathrm{v})$ by heat ing for $30 \mathrm{~min}$ at $130^{\circ} \mathrm{C}$ under nitrogen. After hydrolysis and derivatization, the samples were analyzed by gas chromatography/isotope-dilution mass spec trom etry with selected ion-monitoring according to the method described by Dizdar oglu [17].

A Hewlett Packard Model 5890 Series II Model gas chromatograph interfaced to a Hewlett Packard Model 5972 mass selective detector was used. The injection port and $\mathrm{GC} / \mathrm{MS}$ interface were both maintained at $250^{\circ} \mathrm{C}$ and the ion source at about $200^{\circ} \mathrm{C}$. Separations were car ried out us ing a fused-silica capillary col umn (UI tra 2, $12.5 \mathrm{~m} \times 0.2 \mathrm{~mm}$, Hewlett Packard) coated with cross-linked $5 \%$ phenylmethylsilicone gum phase (film thickness $0.33 \mu \mathrm{m}$ ). An aliquot of each derivatized sam ple $(4 \mu \mathrm{l})$ was in jected with out any fur ther treatment into the injection port of the gas chromatograph by means of an autosampler.

Data processingandsta tisti cal analysis. The DNA re pair curves de scribed by the equa tion $y=a e^{-b t}+c$ were fitted (by the least squaremethod) to the ex per i men tal val ues. In this statistical model a is the reparable damage in duced by ra di a tion, $c$ is the irreparable dam age (to tal dam age isa $+c$ ) and $b(=1 / \tau)$ is the time constant for the repair of that damage, $\tau$ is the time re quired to re duce the rep a ra ble dam age to $37 \%$ of its ini tial amount. The significance of the difference in mean values was es ti mated by the Stu dent's t-test for in de pendent samples. All statistical evaluation and curve fit ting were per formed with the use of Statistica v. 5.1 software (StatSoft Inc. Tulsa, U.S.A.).

\section{RESULTS}

Figure 1 pres ents the ini tial amounts of six types of base damage and their repair in $L Y$ cells that were irradiated with 400 Gy of $\gamma$ rays. The following altered bases were determined: 5-hydroxyuracil (5-OHUra), 5-hydroxymethyluracil (5-OHMeUra), 5-hydroxycytosine (5-OHCyt), 8-hydroxyadenine (8-OHAde), 4,6-diamino-4-hydroxy-5-formamidopyrimidine (FapyAde), and 8-hydroxyguanine (8-OHGua).

Generally, the total amount of initial base dam age was higher in the ra di a tion sen si tive $L Y-S$ subline than in the radiation resistant LY -R subline. Although the products identified in the course of this study were typ i cal for radiation treated chromatin, the amount of base damage reported is higher than that reported in the literature (reviewed in [18]). How ever, it is in ex cel lent agr eement with the previously reported results [13]. The repair rates dif fered be tween sublines and were not related to the initial amount of the given altered base. The data were fitted to the equation $y=a e^{-b t}+c$. The parameters for all repair curves arepresented in Ta ble 1. Al though the re pair rates of the given al tered bases differ between sublines, the re pair rates of the total base dam age (the sum of all al tered bases) were similar in both cell lines (Fig. 2A, Table 1). How ever, if the rel a tive amounts of the 

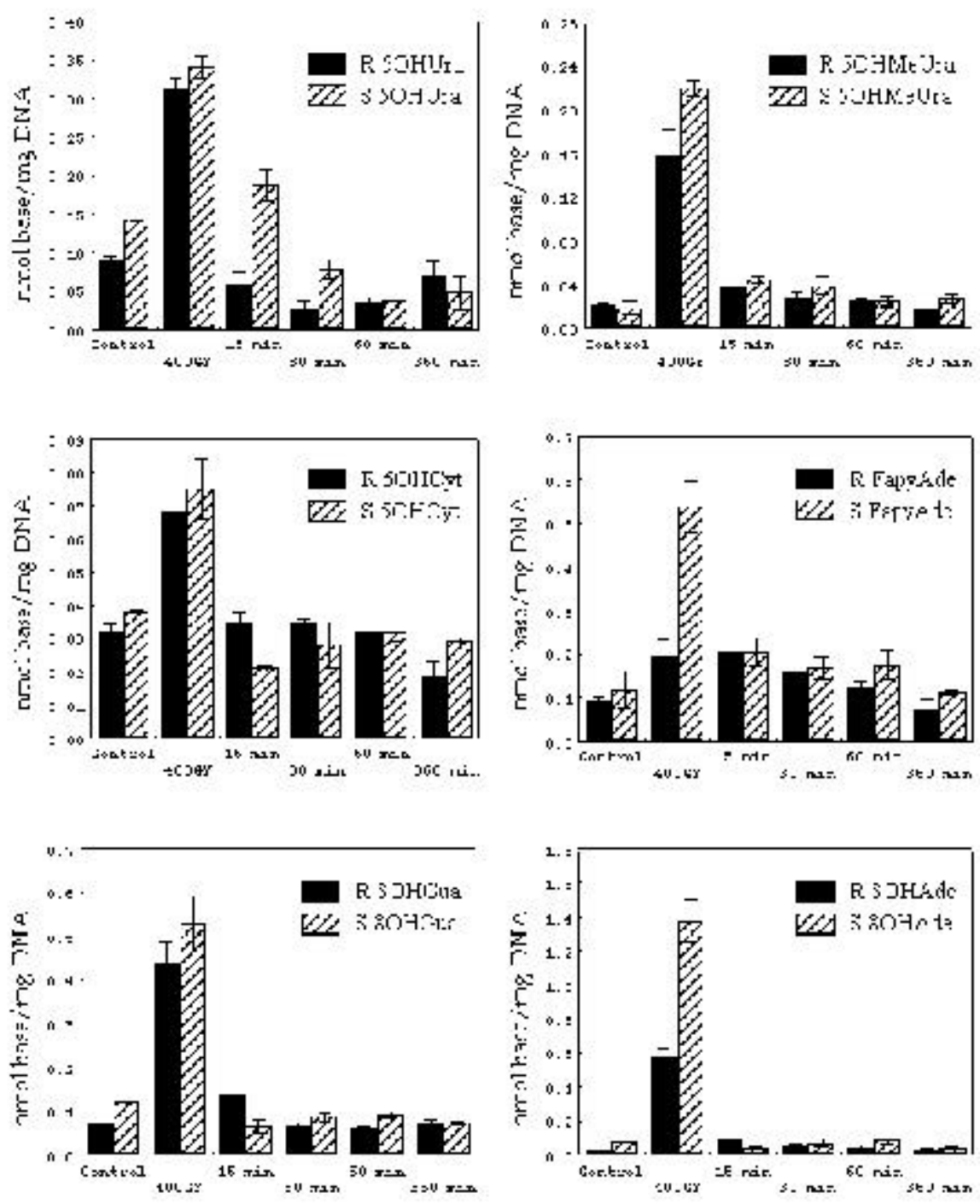

Fig ure 1. Ini tial DNA base dam age and its re pair in $\gamma$-irradiated (400 Gy) LY-R and LY-S cells.

Mean re sults from 3 ex per i ments $\pm S E M$.

total base dam age are plot ted, slower repair in LY-R cells is noticeable (Fig. 2B).

The most marked difference between LY-R and LY-S cells was in the initial amount of FapyAde and in the rate of its removal: the amount was con sid er ably higher in $L Y$-S cells than in LY-R cells, whereas LY-R cells removed it much more slowly than LY-S cells. As shown in Fig. 1, the re pair in LY-S cells was almost completed after $15 \mathrm{~min}$; at that time, no dam age was re paired in LY-R cells. A twofold higher content of 8-OHAde was found upon irradiation in LY-S cells than in LY-R cells, how ever, the rates of re pair did not significantly differ. In the case of 8-OH Gua, the re pair rate and the ini tial amount of the damaged base were higher in LY-S cells than in LY-R cells. As can be seen in Fig. 1, the dif ference in damage removal con cerns mainly the 15 min point. In con trast, $5-\mathrm{OH}$ U ra was gen erated in equal amounts in both sublines, but the repair rate in this case was markedly lower in LY -S than in LY-R cells. To make this com pli cated pat tern eas ier to fol low, we pres ent the dif fer ences between the $L Y$ sublines in a simplified way in Table 2 .

The con trol lev els werestrikingly high in the case of 5-OHUra (LY-S cells) and FapyAde (LY-R cells; cf. Fig. 1). Com paring the con trol lev els and the re pair rates in these cases, one 
can see that where the re pair was slow -the control content was high, a perfectly logical out come. How ever, there was no sta tis ti cally sensitive LY $-S$ subline, its repair rates in the LY sublines seem to be un related to the dif ferential lethal effect of irradiation. The repair

Ta ble 1. Ini tial amounts (a), time con stants of re pair $(\tau)$ and re sid ual amounts (c) of var $i$ ous types of base dam age in LY-R and LY-S cells ir ra di ated with $400 \mathrm{~Gy}$ of $\gamma$-rays

\begin{tabular}{lcccr}
\hline & \multicolumn{3}{c}{ LY-R } & \multicolumn{1}{c}{$c$} \\
\hline 5-OHUra & $\mathrm{a}$ & $\mathrm{b}$ & $\mathrm{c}$ & 6.2 \\
5-OHMeUra & $0.28 \pm 0.02$ & $0.16 \pm 0.143^{1}$ & $0.03 \pm 0.013$ & 7.1 \\
5-OHCyt & $0.13 \pm 0.01^{1}$ & $0.14 \pm 0.033$ & $0.02 \pm 0.004$ & 11.1 \\
FapyAde & $0.04 \pm 0.01$ & $0.09 \pm 0.099$ & $0.03 \pm 0.007$ & 100.0 \\
8-OHAde & $0.13 \pm 0.03^{1}$ & $0.01 \pm 0.006^{1}$ & $0.07 \pm 0.020$ & 6.2 \\
8-OHGua & $0.54 \pm 0.01^{1}$ & $0.16 \pm 0.020$ & $0.04 \pm 0.006$ & 9.1 \\
Total & $0.37 \pm 0.01^{1}$ & $0.11 \pm 0.012^{1}$ & $0.06 \pm 0.007^{1}$ & 3.3 \\
\hline & $1.44 \pm 0.02^{1}$ & $0.11 \pm 0.007$ & $0.30 \pm 0.014^{1}$ & \\
\hline 5-OHUra & $\mathrm{a}$ & $\mathrm{LY}-\mathrm{S}$ & & $\mathrm{c}$ \\
5-OHMeUra & $0.30 \pm 0.02$ & $0.05 \pm 0.01^{1}$ & $0.03 \pm 0.015$ & 20.0 \\
5-OHCyt & $0.19 \pm 0.01^{1}$ & $0.16 \pm 0.04$ & $0.03 \pm 0.004$ & 6.2 \\
FapyAde & $0.04 \pm 0.001$ & $0.22 \pm 0.04$ & $0.03 \pm 0.001$ & 4.5 \\
8-OHAde & $0.39 \pm 0.04^{1}$ & $0.12 \pm 0.05^{1}$ & $0.14 \pm 0.021$ & 8.3 \\
8-OHGua & $1.31 \pm 0.02^{1}$ & $0.29 \pm 0.13$ & $0.06 \pm 0.013$ & 3.4 \\
Total & $0.44 \pm 0.01^{1}$ & $0.25 \pm 0.05^{1}$ & $0.08 \pm 0.004^{1}$ & 4.0 \\
\hline
\end{tabular}

Equation $y=a e^{-b t}+c$ wasfit ted to theex per i mental val ues; $a$ is themea sure of therep a rabledam agein duced by ra di ation; $c$ is the ir rep a ra bledam age (to tal dam ageis $a+c)$ and $b(=1 / \tau)$ is the time con stant for the re pair of that dam age; $\tau$ is the time required to reducetherep a ra ble dam ageto $37 \%$ of its ini tial amount. Results rep resent est i mated value \pm S.E. ${ }^{1}$ Significantdifference, $L Y-R$ versus $L Y-S$.

significant correlation between the control base dam age lev els and the val ues of $b$, when the data for all types of base dam age were considered.

\section{DISCUSSION}

The yield of DNA base damage induced by low-LET ionizing radiation has been estimated to be 2.7 times the yield of sin gle strand break, that is, 2700 damaged bases per cell per $\mathrm{Gy}$. This type of damage seems unimportant for thelethal ef fect of ir ra di a tion in mammalian cells (reviewed in [19]). Although the base damage is higher in the more radio- rates of various types of base damage in the radiation sensitive LY-S cell line are either equal to those in the radioresistant counterpart, LY-R, or higher (Tables 1 and 2), with one exception (5-OHUra). In spite of irradiation with a very high dose (400 Gy), about $80 \%$ of dam age usu ally is removed dur ing the first 15 min, as can be seen in Fig. 1.

The most striking difference between LY-R and LY $-S$ cells found in this study was in the initial amount of FapyAde. This difference may be due to the enhanced in duction of the damage or to the enhanced rate of its removal. The former is rather unlikely in the case of L5178Y cells, since the amount of F apyA de is con sid er ably higher in LY -S cells, 
but its removal is slower in LY-R cells. Whether these two closely related cell lines can differ so much in the induction of initial polymerases [2], is 12 times lower in the radioresistant $L Y-R$ subline than in the radiosensitive $L Y-S$ subline.

Ta ble 2. DNA base dam age (ini tial amount and re pair rate) in LY cells $\gamma$-irradiated with $400 \mathrm{~Gy}$

\begin{tabular}{lccc}
\hline \multirow{2}{*}{ Damaged base } & Initial amount & Repair rate & Residual damage \\
\cline { 2 - 4 } & & LY-R versus LY-S & \\
\hline 5-OHUra & Equal & Higher & Equal \\
5-OHMeUra & Lower & Equal & Equal \\
5-OHCyt & Equal & Equal & Equal \\
FapyAde & Lower & L ower & Equal \\
8-OHAde & Lower & Equal & Equal \\
8-OHGua & Lower & L ower & L ower \\
Total & Lower & Equal & L ower \\
\hline
\end{tabular}

DNA damage needs to be further clarified. The potential factors that can preferentially mod ify thein duc tion of DNA base dam ageare intracellular redox environment and transition metal ion content [20-23].

Al though the dam aged bases seem to be very ef fi ciently removed, their location in thevi cin ity of other le sions gives rise to mul ti ply damaged sites, and thus adds to the lethal ef fect of irradiation [19]. The delay in repair of such sites may be the ul ti mate death cause: as suggested by Aldridge and Radford [24], the time period avail ablefor DNA repair prior to potential activation of apoptosis is a critical determinant of radiosensitivity in some cell lines. Thus, base damagemay in di rectly con tribute to the overall lethal effect of radiation. Estimation of this contribution would be rather dif fi cult with out ap ply ing a much moresen sitive analytical method. However, judging from the data on base dam age re pair in the $L Y$ sublines (Ta bles 1 and 2), the rate of re pair is not related to the radiation sensitivity. This result does not support the assumption that DSB gen er ation dueto cluster ed basedam age excision contributes to the delayed rejoining of DSB in LY-S cells; the delayed DSB rejoining ob vi ously is caused by a de fect in the function ing of the DSB repair sys tem. Even there moval rate of the potentially lethal formamidopyrimidine that effectively blocks DNA
The role of base dam age in mu ta gen esis is a matter of debate $[2,19,25]$. There is strong ev i dence that mul ti ply dam aged sites are the causativelesionsin muta gen esis (reviewed in [19]). On the other hand, oxidized bases are
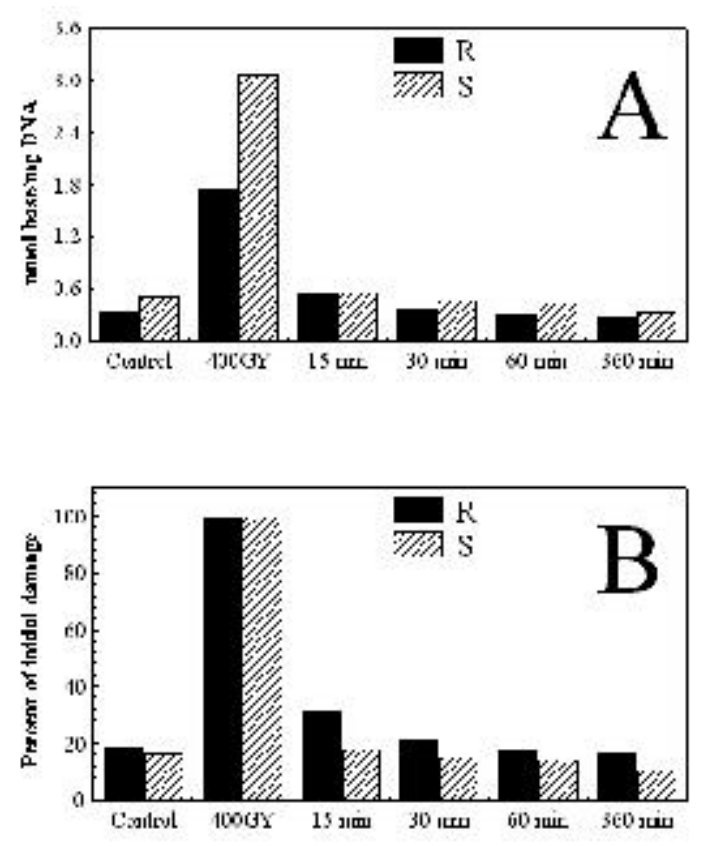

Figure 2. To tal ini tial DNA base dam age (sum of all altered bases) and its repair in $\gamma$-irradiated (400 Gy) LY-R and LY-S cells expressed as the amount of al tered bases (A) or the per cent age of the ini tial dam age (taken as $100 \%$ ) (B).

abundant in cellular DNA and are implicated in var i ous patho log i cal pro cesses $[26,27]$ and 
ageing [28]. In bacteria, baseexcision repair enzymes are anti-mutagenic, as indicated by enhanced mutation frequencies in strains defec tivein theac tiv ity of glycosylases in volved in the repair of oxidised bases [25]. Whether there is an analogy between bacteria and mammalian cells, remains to be seen when suitable mu tant mam ma lian cell lines are obtained.

Since the lo cally mul ti ply dam aged sites also comprise base damage, the relative amounts of base damage and strand breaks and their repair rates, especially under conditions of low doserateir ra di a tion, may af fect theyield of le thal or mutagenic le sions. In this respect, the $L Y$ sublines fit this general pattern. The higher level of radiation induced DNA base damage in LY-S cells is compensated by higher rates of repair of thepo ten tially lethal formamidopyrimidine, and of the highly mutagenic 8-OH Gua (the lat ter seen when the percentage of damage at the $15 \mathrm{~min}$ time point is com pared in the LY sublines, Fig. 2B). However, since only about $30 \%$ of $X$ ray induced mutations are point mutations [2] the higher rates of repair of FapyAde and 8-OH Gua do not sufficiently explain the hypomutability of $L Y$-S cells ex posed to ion iz ing ra diation [3, 7]; hence, other cellular processes may be responsible for the low mutability of these cells. The remaining $70 \%$ of $X$ ray induced mutations are deletions and chromosomal rearrangements; if it happens that the tar get lo cus is in a close vi cin ity to that of essen tial genes -their loss causes cell kill, thus excluding mutations in the target locus from the analysis and resulting in an apparent hypomutability (as proposed by Evans to explain the hypomutability of LY-S cells [29, 30]).

In summary, the repair rates of the $\gamma$-ray-induced DNA base damage in the LY sublines are related neither to the initial amounts of the dam aged bases nor to the differential lethal or mutagenic effects of ionizing radiation in these sublines. Although there is no doubt that the im pair ment of DSB rejoining is the main cause of LY $-S$ susceptibil ity to ion izing ra di ation [8], our result does not sup port the as sump tion that DSB gen er a tion dueto ex ci sion of clus tered base dam age con tributes to the delayed rejoin ing of DSB in these cells.

We are grateful to Dr. Miral Dizdaroglu from the National In sti tute of Stan dards and Technology (Gaithersburg, MD, U.S.A.) for the stable isotopela belled in ter nal stan dards used in this work. We appreciate the expert assistance of Dr. Teresa Wroñska (INCT, War szawa) in the MINEOLA $\gamma$-ray source operation.

\section{REFERE N CES}

1. Ward, J.F. (1999) Na ture of lesions formed by ionizing radiation; in DNA Damage and Repair, vol 2: DNA Repair in Higher Eucaryotes (Nickoloff, J.A. \& Hoekstra, M.F., eds.) pp. 65- 84, Humana Press, Totowa, New J ersey.

2. Wallace, S.S. (1998) En zy matic pro cess ing of radiation-induced free radical damage in DNA. Radiat. Res. 150, S60-S79.

3. Beer, J.Z., Budzicka, E., Niepokojczycka, E., Rosiek, O., Szumiel, I. \& Walicka, M. (1983) Loss of tumorigenicity with simultaneous changes in radiosensitivity and photosensitivity during in vitro growth of L5178Y murine lymphoma cells. Cancer Res. 43, 4736- 4742.

4. Bouzyk, E., Buraczewska, I., Rosiek, O., Sochanowicz, B. \& Szumiel, I. (1991) Hydrogen per ox ide in duced repro duc tiveand interphase death in two strains of L5178Y murine lymphoma differing in radiation sensitivity. Radiat. En vi ron. Biophys. 30, 105- 116.

5. Bouzyk, E., Iwanenko, T., Jarocewicz, N., Kruszewski, M., Sochanowicz, B. \& Szumiel, I. (1997)Anti oxi dant defensesystem in differ entially hydrogen peroxide sensitive L5178Y sublines. Free Radical Biol. M ed.22, 697- 704. 
6. Kruszewski, M., Green, M.H., Lowe, J.E. \& Szumiel, I. (1994) DNA strand breakage, cytotoxicity and mutagenicity of hydrogen per oxidetreatmentat $4^{\circ} \mathrm{C}$ and $37^{\circ} \mathrm{C}$ in L5178Y sublines. Mutat. Res. 308, 233- 241.

7. Kruszewski, M., Green, M.H., Lowe, J.E. \& Szumiel, I. (1995) Comparison of effects of iron and cal cium chelators on the re sponse of L5178Y sublines to X-rays and $\mathrm{H}_{2} \mathrm{O}_{2}$. Mutat. Res. 326, 155- 163.

8. W lodek, D. \& Hittelman, W .N. (1987) The repair of double-strand DNA breaks correlates with radiosensitivity of L5178Y-S and L5178Y-R cells. Radiat. Res. 112, 146- 155.

9. W lodek, D. \& Hittelman, W.N. (1988) The relation ship of DNA and chro mo somedam ageto sur vival of syn chro nized X-irradiated L $5178 Y$ cells. II. Re pair. Radiat. Res. 115, 566-575.

10. W lodek, D. \& Hittelman, W.N. (1988) The relation ship of DNA and chro mo somedam ageto sur vival of syn chro nized X-irradiated L 5178Y cells. I. Initial damage. Radiat. Res. 115, 550- 565.

11. Kapiszewska, M., Szumiel, I. \& Lange, C.S. (1992) Damage at two levels of DNA folding measured by fluorescent halo technique in X-irradiated L5178Y R and L5178Y -S cells. I. Initial lesions. Radiat. Environ. Biophys. 31, 311- 322.

12.Szumiel, I., Kapiszewska, M., Kruszewski, M., I wanenko, T. \& Lange, C.S. (1995) Con tent of iron and cop per in thenu clei and in duction of $\mathrm{pH}$ 9-abile lesions in L5178Y sublines inversely cross-sensitive to $\mathrm{H}_{2} \mathrm{O}_{2}$ and $\mathrm{X}$-rays. Radiat. En vi ron. Biophys. 34, 113- 119.

13.Zastawny, T.H., Kruszewski, M. \& Olinski, R. (1998) I on izing radi ation and hy drogen per oxide induced oxidative DNA base damage in two L5178Y cell lines. Free Radical Biol. Med. 24, 1250- 1255 .

14. Mori, T. \& Dizdaroglu, M. (1994) I on izing ra dia tion causes greater DNA base dam age in ra diation-sensitive mutant M 10 cells than in par- ent mouse lymphoma L5178Y cells. Radiat. Res. 140, 85- 90.

15. Szumiel, I. (1979) Re sponse of two strains of L5178Y cells to cis-dichlorobis (cyclopentylamine)plat i num(II). I. Cross-sensitivity to cisPAD and UV light. Chem.-Biol. Interact. 24, 51- 72 .

16. Mee, L.K. \& Adelstein, S.J . (1981) Predomi nance of core his tones in for ma tion of DNAprotein crosslinks in gamma-irradiated chromatin. Proc. Natl. A cad. Sci. U.S.A. 78, 21942198.

17. Dizdaroglu, M. (1994) Chemical determina tion of ox i da tive DNA dam age by gas chro ma tography-mass spectrometry. M ethods Enzymol. 234, 3- 16.

18. Halliwell, B. \& Dizdaroglu, M. (1992) The measurement of oxidative damage to DNA by HPLC and GC/MS tech niques. Free Radical Res. Commun. 16, 75-87.

19. W ard,J .F. (1995) Radi a tion mu ta gen esis: The initial DNA lesions responsible. Radiat. Res. 142, 362- 368.

20.Dizdaroglu, M., Rao, G., Halliwell, B. \& Gajewski, E. (1991) Dam age to the DNA bases in mammalian chromatin by hydrogen per ox ide in the presence of fer ric and cu pricions. Arch. Biochem. Biophys. 285, 317- 324.

21.Gajewski, E., Rao, G., Nackerdien, Z. \& Dizdar oglu, M. (1990) M od i fi cation of DNA bases in mammalian chromatin by radiation-generated free radicals. Biochemistry 29, 7876- 7882.

22.Mar tins, E.A., Chubatsu, L.S. \& Meneghini, R. (1991)R ole of an ti oxi dantsin protecting cel lur lar DNA from damage by oxidative stress. Mutat. Res. 250, 95- 101.

23. Meneghini, R. (1997) I ron ho meo sta sis, ox i da tive stress, and DNA damage. Free Radical Biol. M ed. 23, 783- 792.

24.Aldridge, D.R. \& Radford, I.R. (1998) Explaining differ ences in sen si tiv ity to kill ing by 
ionizing radiation between human lymphoid cell lines. Cancer Res. 58, 2817- 2824.

25.Laval, J., J urado, J ., Saparbaev, M. \& Sidorkina, O. (1998) Antimutagenic role of baseexcision repair enzymes upon free radical-induced DNA damage. Mutat. Res. 402, 93- 102.

26.Beckman, K.B.\& Ames, B.N. (1997) Ox i da tive decay of DNA. J. Biol. Chem. 272, 1963319636.

27. Loft, S. \& Poulsen, H.E. (1996) Cancer risk and oxidative DNA damage in man. J. Mol. Med. 74, 297- 312.
28.Bohr, V.A. \& Anson, R.M. (1995) DNA damage, mu ta tion and fine struc ture DNA re pair in aging. Mutat. Res. 338, 25- 34.

29.Ev ans, H.H., Ricanati, M., Di Salvo, C., Horng, M.F. \& Mencl, J. (1991) Induction of multilocus le sions by UVC-radiation in mouse L5178Y lymphoblasts. Mutation Res. 251, 79- 90.

30.Yi, P.N., Evans, H.H., Beer, J .Z. \& Rha, C.K. (1994) Relationships between mitotic delay and the dose rate of $X$ ra di a tion. Radiat. Res. 140, 387- 392. 